\title{
Hydrothermal vents or hydrothermal fields: Where can life begin?
}

\author{
DAVE DEAMER
}

Because salty seawater dominates the Earth's surface today, it is natural to think that life began in the ocean, perhaps in hydrothermal vents. However, from a biophysical perspective there are significant limitations to this conjecture. An alternative site is hydrothermal fresh water. Volcanic regions of Kamchatka, Iceland and Hawaii have abundant fresh water pools that undergo cycles of hydration (precipitation) and dehydration (evaporation). We have prepared a laboratory simulation of such cycles using solutions of mononucleotides mixed with lipids that can assemble into membranous structures. When the mixtures were exposed to multiple wet-dry cycles, RNA-like polymers ranging from 10 to over 100 nucleotides in length were products. Furthermore, in the final hydration phase the polymers are encapsulated in lipid vesicles to form protocells. We propose that robust protocells composed of encapsulated RNA-like polymers represent the first step toward the origin of life in a fresh water environment. Our attempts to reproduce these observations in seawater failed. These results have obvious implications related to the search for life on Mars and icy moons like Enceladus and Europa. 